\title{
Von «der» Planung zu multiplen Planungen: Strategische Entscheidungen unter Bedingungen hoher Komplexität und geringer Umweltkontrolle
}

\section{Oliver Ibert, Bonn}

\section{Das Problem der Strategiefähigkeit}

Dieser Beitrag beschäftigt sich mit der Frage, wie öffentlich-rechtliche Planung «strategisch» agieren kann. LuHMANN hat Planung kurz als «über Entscheidungen entscheiden» (1971: 67) definiert. Planung meint dem zu Folge eine Sequenz von Entscheidungen und das Reflexivwerden des Entscheidungsprozesses. Sequentiell bedeutet, dass Entscheidungsprozesse zerlegt werden in rational ausgearbeitete «Entscheidungsprämissen» und fallspezifische, handlungsbezogene «operative» (FAludi 1985; MAYER, S. 1999) Entscheidungen. Reflexiv bedeutet, dass beim Planen (mindestens) zweimal entschieden wird, wobei operationales Entscheiden auf der Basis der Entscheidungsprämissen stattfindet. Im Idealfall ergänzen beide Typen von Entscheidungen einander. Planung ermöglicht Entscheidungsprozesse, die sowohl schnell und reaktiv sind als auch auf hohem Rationalitätsniveau operieren (Faludi 1985). In der Planungspraxis verwischt diese idealtypische sequentielle Arbeitsteilung jedoch, es ergeben sich Pfadabhängigkeiten durch Rückkopplungen (FunKe 1974: 21). Bereits bei der Formulierung von Entscheidungsprämissen verändert sich die Planungsgrundlage durch fortlaufende operative Entscheidungen. Sie schaffen Fakten, die unbeabsichtigt wie Planungsprämissen wirken. Von Planungsstrategie soll hier gesprochen werden, wenn viele Entscheidungen über einen längeren Zeitraum ein «konsistentes Muster» (WiechmanN \& HutTer 2008) herausbilden und so zu einem gewünschten, nicht anders erreichbaren Ergebnis führen.

Bereits die etymologische Wurzel (griechisch strategós $=$ General) deutet an, dass die Idee einer Strategie auf einer impliziten Unterstellung beruht. Eine Strategie kann es eigentlich nur geben, wenn auch ein Akteur existiert, der langfristig nach einer übergreifenden Logik vorgehen kann oder will.

«Dabei sind Kontinuität und Integrität des Akteurs mit umso größerer Selbstverständlichkeit vorausgesetzt, je «strategischer〉 die maßgebenden Handlungsorientierungen erscheinen» (WIESENTHAL 1990: 1).

Um Strategiefähigkeit besser denken zu können, schafft sich die Planungstheorie ein epistemisches Hilfskonstrukt, die Annahme, es gebe ein planendes Subjekt, um eine Projektionsfläche zu erhalten, auf der Strategiefähigkeit besser abgebildet werden kann.
Derartige theoretische Hilfskonstrukte werden allerdings immer dann kontraproduktiv, wenn die durch sie vorgenommene Vereinfachung der Realität in Vergessenheit gerät. Genau das kritisiert Klaus SELLE (2005) am gegenwärtigen planungstheoretischen Diskurs, in welchem zu häufig unkritisch von «der» Planung gesprochen werde, ganz, als ob damit ein Akteur gemeint sei. Das Hilfskonstrukt eines planenden Subjekts ist aber für den heutigen Diskurs um Strategiefähigkeit der Planung aus einem zweiten Grund viel problematischer. Ein in sich widerspruchsfreier Akteur ist nämlich vor allem in Situationen strategiefähig, die übersichtlich und kontrollierbar sind (Wiesenthal 1990) - der General überblickt das Schlachtfeld, kaum aber die großpolitische Lage. In einer Umwelt, die durch Komplexität und Dynamik gekennzeichnet und die durch eigenes Entscheiden nur unvollständig kontrollierbar ist, führen interne Kohärenz und Stabilität zu Entscheidungsketten, die Gefahr laufen, in Sackgassen zu enden.

Der jüngere Governance-Diskurs jedoch macht mehr als deutlich, dass Planungsstrategien unter Bedingungen hoher Komplexität und geringer Umweltkontrolle greifen müssen und am dringendsten benötigt werden: Schon innerhalb der öffentlichen Sphäre fallen raumrelevante Entscheidungen wenig koordiniert in einer Vielzahl von Ämtern, Abteilungen und Hierarchieebenen. Wird das Feld privater und zivilgesellschaftlicher Akteure (SELLE 2005) mitgedacht, potenziert sich die Komplexität. Zudem ist öffentlich-rechtliche Planung durch ein Umsetzungsdilemma gekennzeichnet, privat(wirtschaftlich)e Akteure, nicht die planenden Instanzen, sind die ausführenden Akteure (MaYer, S. 1999; Selle 2005). Rechtliche Regelungen zur Steuerung der privat(wirtschaftlich)en Umsetzung stoßen zunehmend an Komplexitätsgrenzen. Geld, um Akteure am «goldenen Zügel» führen zu können, steht immer weniger zur Verfügung.

Es geht in diesem Artikel darum, die Idee von Strategiefähigkeit in der Planung so weiter zu entwickeln, dass sie den Anforderungen besser gerecht wird, die sich durch Komplexität, Dynamik sowie begrenzte Umweltkontrolle stellen. Unter diesen Bedingungen müssen Planungsstrategien nach unterschiedlichen, sogar widersprüchlichen Logiken vorgehen können (SIEBEL 2006). An die Stelle eines kohärenten und stabilen Planungssubjekts treten diverse Rollenmodelle von Planung, die je eine spezifische Spielart von Strategiefähigkeit repräsentieren. 
Die Argumentation verläuft wie folgt: Zuerst werden Planungstheorien im Hinblick auf Bezugspunkte für strategisches Handeln untersucht. Daraus werden vier Rollenmodelle abgeleitet, und für jedes Modell gezeigt, welchen Aspekt von Strategiefähigkeit es in den Vordergrund rückt, wie es Entscheidungsprozesse strukturiert, und welche spezifischen Stärken und Schwächen es kennzeichnet. Die abschließende Diskussion lotet die Grenzen einer so verstandenen Strategiefähigkeit aus.

\section{Fallbeispiele}

Die Modelle werden aus der Literatur abgeleitet und anhand zweier Fallbeispiele empirisch illustriert, für die Strategiefähigkeit unverzichtbar war. Die Fallbeispiele stammen aus dem Kontext der Internationalen Bauausstellung (IBA) Emscher Park, einem Entwicklungsprogramm des Landes Nordrhein-Westfalen für das nördliche Ruhrgebiet, das zwischen 1990 und 1999 in insgesamt über 100 Projekten zu diversen Themen exemplarische Lösungen zum Strukturwandel altindustrieller Regionen gefördert hat. Mit der IBA-Planungsgesellschaft hat die Landesregierung eine als $\mathrm{GmbH}$ organisierte Innovationsagentur in der Region installiert, die an allen diesen Projekten inhaltlich beteiligt war.

Das erste Beispiel ist ein Wohnprojekt in Recklinghausen-Süd, das speziell die Bedürfnisse allein erziehender Eltern berücksichtigt. Es gilt als ein IBA-Projekt der «ersten Stunde», das in einem ersten Bauabschnitt von 1990 bis 1994 entwickelt und durch einen zweiten Bauabschnitt 1998 ergänzt wurde. Es umfasst insgesamt 47 Wohneinheiten und füllt eine relativ unscheinbare Baulücke an einer südlichen Ausfallstraße Recklinghausens. Wie bei der IBA typisch, waren drei Akteursgruppen zentral in einer operativen Projektgruppe versammelt: die lokale Wohnungsbaugesellschaft als Grundbesitzerin, das Stadtplanungsamt und ein Vertreter der IBA-Planungsgesellschaft. Das Projekt gilt als hoch ambitioniert. Es schafft nicht nur für allein erziehende Eltern adäquate bauliche Strukturen, sondern bezieht über einen Partizipationsprozess die zukünftigen Nutzerinnen und Nutzer bei der Gestaltung ein. Deren soziales Zusammenleben in der Siedlung soll dadurch in Richtung einer intensivierten gegenseitigen nachbarschaftlichen Hilfe beeinflusst werden.

Das zweite Fallbeispiel betrifft eine gewerbepolitische Maßnahme zur Revitalisierung der ehemaligen Zeche Erin in Castrop-Rauxel. Es galt, eine mit Altlasten verseuchte und altindustriellen Ruinen vorstrukturierte Fläche in einen hochwertigen Gewerbepark mit positivem Image zu verwandeln. Das Projekt umfasste die volle Laufzeit der IBA. Auch hier gab es eine operative Projektgruppe, in der die damals landeseigene
Entwicklungsgesellschaft (LEG) als Grundbesitzerin, die Stadt Castrop-Rauxel durch die Wirtschaftsförderung sowie die IBA-Planungsgesellschaft repräsentiert waren. Für die krisengebeutelte Stadt ist die 40 Hektar große Fläche von herausragender Bedeutung. Sie liegt gleich neben der Innenstadt und bietet die größte Aussicht, zukunftsfähige Arbeitsplätze anzusiedeln. Eine Besonderheit dieses Projektes war ein im Hintergrund der Projektentwicklung förderlich wirkender informeller «Patenkreis» aus bis zu 30 einflussreichen Akteuren aus Landes- und Kommunalpolitik, Verwaltung, Wirtschaftskammern und Banken.

Die Fallbeispiele wurden ausgewählt, weil ihr Innovationsanspruch hoch war, und sie damit gezwungen waren, unter Bedingungen großer externer Unsicherheit ein hohes Maß an Strategiefähigkeit zu entwickeln. Ein Vergleich beider Fälle lohnt, weil ihre Besonderheiten (Partizipationsprozess und Patenkreis) jeweils andere Aspekte von Planungsrationalität stärker betonen. Als Datengrundlage dienen 17 Experteninterviews mit Projektbeteiligten sowie Sekundärmaterialien zu den Vorhaben. Die Daten wurden im Zuge eines begleitenden Forschungsprojektes (IBERT, MAYER \& SIEBEL 2002) zwischen 1999 und 2001 erhoben.

\section{Ambiguität von Planung}

\subsection{Politische vs. fachliche Aufgabe}

Die Funktion, Entscheidungsprämissen für Dritte festzulegen, versetzt Planung in ein Spannungsfeld, sie lässt sich nicht eindeutig der Politik oder dem rein «fachlichen» Verwalten zuordnen (BAUHARDT 2004; LUHMANN 1971). Das synoptische Ideal von Planung betont ihren fachlichen Charakter. Planung schwebt gleichsam über interessengeleiteten politischen Streitereien (GANs 1969). Ihre Legitimität beruht auf einer klaren Arbeitsteilung, in der Politik beauftragt und kontrolliert, die Verwaltung politische Vorgaben in fachlich begründete Handlungsalternativen operationalisiert und in verwaltungstechnische Beschlüsse umsetzt (LUHMANN 1971). Das inkrementalistische Modell sieht Planungsergebnisse hingegen als gut an, wenn die Betroffenen sich damit einverstanden erklären können. Der pluralistisch ausgehandelte Konsens wird zum Gradmesser für Planungsrationalität. Da in der Praxis aber Pluralismus durch Machtunterschiede überlagert wird, dringt der politische Charakter von Planung direkt in Prozesse der Entscheidungsfindung ein. Die Interessen mächtiger Gesellschaftsgruppen werden bei der Formulierung und Abwägung von Handlungsalternativen von vornherein berücksichtigt (BRAYBROOKE \& LindBLOM 1972).

\subsection{Verstetigung vs. Revidierbarkeit}

Die Charakterisierung von Planung als Handlungsvorbereitung lässt die Frage in den Vordergrund treten, 


\begin{tabular}{|l|ll|}
\hline Planung & als fachliche... & und politische Aufgabe \\
\hline Verstetigung & INGENIEUR & KOOPER ATIONSPARTNER \\
& wissenschaftlich- & politische Rationalität \\
technische Rationalität & \\
Revidierbarkeit & handlungsorientierte & diskursive Rationalität \\
& Rationalität & \\
\hline
\end{tabular}

Tab. 1: Multiple Identitäten öffentlich-rechtlicher Planung

Multiple identities in public planning

Identités multiples de la planification publique

was höher zu gewichten sei, die langfristige Verstetigung oder die flexible Revidierbarkeit einmal getroffener Entscheidungen (FALUdi 1986; FunKe 1974; WiechMANn \& HUTTER 2008). Verstetigung bedeutet, die Wirkung von Planung bemesse sich daran, inwieweit einmal festgelegte Entscheidungsprämissen kontinuierlich fortgeführt würden. Operative Entscheidungen sollen möglichst wenig von formulierten Prämissen abweichen. Es gilt,

«durch die Minimierung des Ermessensspielraums willkürliche Entscheidungen zu verhindern» (MAYER, S. 1999: 161).

Das Prinzip der Revidierbarkeit betont dagegen Anforderungen wie Flexibilität, Fehlertoleranz, Adaptivität und Freiheit für dezentrale Entscheidungen. Es geht darum, sich nicht zum Gefangenen früherer Entscheidungen zu machen. Pläne werden primär als «Ressource» (Suchman 1987) betrachtet, die beim situativen Reagieren erlauben, günstige Gelegenheiten zu erkennen und zu nutzen (RoBERTs 2002).

\section{Rollenauslegungen}

Das Spannungsfeld zwischen fachlichem und politischem Verständnis, Verstetigung und Revidierbarkeit bietet Orientierungen für strategische Planung (Tab. 1).

\subsection{Ingenieur: Planung als technizistische Problemlösung}

Die Ingenieursrolle verschneidet das Selbstverständnis des Experten für Problemlösungen mit einer Aus- richtung von Planung zugunsten eines hohen Grads an Verstetigung. Idealtypisch entscheidet der oder die Ingenieur(in) in fachlicher Objektivität jenseits politischer Interessenkonflikte und formuliert Entscheidungsprämissen für operative Entscheidungen bis ins Detail bindend. Die Formulierung von Entscheidungsprämissen wird durch die planende Verwaltung vorgenommen, operative und handlungsbezogene Entscheidungen durch private Raumnutzer und Investoren getroffen, wobei letztere die Intentionen des Plans gleichsam stellvertretend im praktischen Handeln umsetzen. Dahinter steckt ein wissenschaftlich-technisches Rationalitätskonzept. Planerische Vorentscheidungen sollten dem Anspruch nach dem Vorbild wissenschaftlicher Analyse folgen und ihre Gegenstände systematisch erforschen sowie die richtigen Planungsziele logisch ableiten (STORBECK 1969; kritisch: FUNKE 1974; RITTEL 1992). Sind die Entscheidungsprämissen sorgfältig durchdacht und die Instrumente in ihren Steuerungswirkungen vollkommen verstanden, dann erscheinen die nachfolgenden Schritte bloß noch als Vollzug der im Plan angelegten Intention.

Die Ingenieursrolle ist für eine Organisation von Innovation an zwei Stellen produktiv. Erstens, beruht jedwede Planung auf einem «moderaten Optimismus» (RitTel 1992), und die Haltung der Ingenieurin oder des Ingenieurs hält die Erfahrung der erfolgreichen rationalen Problembearbeitung bereit. Das rechtfertigt etwa das Zutrauen von Planerinnen und Planern, das soziale Zusammenleben in einer Siedlung nicht nur 
durch bauliche Maßnahmen zu ermöglichen, sondern, wie im Wohnprojekt Recklinghausen-Süd, gegenseitige nachbarschaftliche Unterstützung regelrecht sozial zu «konstruieren» (FARWER 2002). Die späteren Bewohner wurden aus einer breiteren Interessentengruppe individuell geprüft und für das Projekt nach individueller Eignung «handverlesen». Zudem wurden bewusst allein erziehende Eltern (wenig Zeit, Nachbarschaftsorientierung) und ältere Menschen (viel Zeit, häufig Kontaktarmut, Nachbarschaftsorientierung) in einem Wohnkomplex zusammengeführt, so dass sich deren spezifische Ressourcen komplementieren und eine intensivierte Nachbarschaftshilfe ermöglichen.

Zweitens bedeutet Innovation nicht allein die Entwicklung ungewohnter Ideen, sondern auch deren praktische Umsetzung, das Tun (IBERT 2003). Jede noch so neuartige Lösung muss in lösbare Einzelaufgaben heruntergebrochen werden. Am Gewerbepark Erin wird deutlich, dass an bestimmten Stellen im Planungsprozess schlicht und einfach solide und durchkalkulierte Problemlösungen gebraucht werden. Dort drohte das ungelöste technische, finanzielle und rechtliche Problem der Sanierung vergifteter Böden auf einer Industriebrache strukturpolitisch motivierte Überlegungen im Keim zu ersticken. Die Aufgabe wurde daraufhin an einen «Arbeitskreis Altlasten» delegiert, dessen Lösungsvorschlag (Aufschüttung und Einkapselung) die Grundlage für den weiteren Prozess bildete. Für diese partielle, eher technische als soziale Problemlösung griff die Ingenieursrolle. Die eingegrenzte Aufgabe konnte unter vollständiger Information, umfassendem Problemverständnis und eindeutiger Zielsetzung gelöst werden.

So hilfreich die Planungsrationalität des Ingenieurs ist, um ein bereits eingegrenztes Teilproblem abschlieBend zu lösen, so hinderlich wird sie, wenn es darum geht, das zentrale Problem zu definieren oder gar einen neuen normativen Bewertungskontext für Problemlösungen zu entwickeln (RitTel 1992). Die technische Problemlösung im Umgang mit giftigen Böden beim Gewerbepark Erin beruht auf einer sozialen Neudefinition, die sich der Ingenieurslogik entzieht. Statt wie bisher das Problem entweder öffentlich herunterzuspielen oder aber es räumlich zu verdrängen, indem die Böden komplett ausgehoben und abtransportiert wurden, wurde hier das Problem offensiv eingestanden, auf der Fläche bearbeitet und auch noch symbolisch durch eine Landschaftsarchitektur überhöht, die die Künstlichkeit der entstehenden Abraumhalden betont.

\section{2 (Stadt-)Manager: Planung als Organisations- entwicklung}

Planerinnen und Planer in der Rolle als (Stadt-)Manager interpretieren ihre Tätigkeiten analog zum Inge- nieur primär unpolitisch (BAUHARDT 2004: 32), richten jedoch ihr Augenmerk verstärkt auf situative und revidierbare operative Entscheidungen. Die Formulierung von Entscheidungsprämissen wird hier von der öffentlichen Verwaltung an eine Organisation übertragen, die privatwirtschaftlich handeln kann, etwa eine städtische Entwicklungsgesellschaft. Entscheidungsprämissen beziehen sich hier nicht mehr auf die Entscheidungen Dritter, sondern auf die zukünftigen operationalen Entscheidungen dieser oft neu gegründeten Organisation. Dem Rollenverständnis liegt eine handlungsorientierte Rationalität zugrunde. Planung könne gesellschaftlichen Nutzen nur stiften, wenn das Vorgedachte auch umgesetzt werde (FALUdi 1985; SINNING 2006).

Die Vorteile sind evident. Wenn sich kein privater Investor findet, um den Plan lebendig werden zu lassen, dann schlüpfen Planer eben selber in diese Rolle. Beide Projekte, Erin und Recklinghausen-Süd, haben, wie dargestellt, das Projektmanagement an eine operative Projektgruppe außerhalb der öffentlichen Verwaltung delegiert, die jeweils einen Vertreter der Kommune, des Grundbesitzers sowie der Internationalen Bauausstellung umfasste. Diese Projektgruppen haben nicht primär Pläne ausgearbeitet, sondern die Entwicklung und Vermarktung der Flächen gesteuert. Im Gewerbepark Erin haben zudem öffentlich beeinflusste Investoren eine Anstoßfunktion übernommen. Sie haben als Erste ihre Vorhaben auf der Fläche realisiert und damit die Entwicklung angestoßen. Zudem haben sie die Qualitätsansprüche des neuartigen Gewerbeparks exemplarisch umgesetzt und so nachfolgende private Investoren «genötigt», sich in ihren Investitionsentscheidungen an ihnen zu orientieren.

Planung als Management erschließt spezifische strategische Optionen für eine Organisation von Innovation, etwa weniger formale Hürden, um die gewünschten Spezialisten zusammenzuführen, sich im Zweifel zugunsten qualitativ hochwertiger Lösungen zu entscheiden oder innovationsspezifische Risiken finanziell abzufedern. Auch die erwähnte IBA-Planungsgesellschaft, die als privatrechtliche $\mathrm{GmbH}$ organisierte Innovationsagentur im Besitz der Landesregierung Nordrhein-Westfalen, verfügte über, zwar bescheidene, aber inhaltlich ungebundene finanzielle Mittel, die sie punktuell in innovationsfördernde Maßnahmen einfließen lassen konnte.

Die wichtigsten Grenzen handlungsorientierter Rationalität liegen darin begründet, dass Planer sich als Handelnde in eine Konstellation begeben, in der sie einerseits nicht privatwirtschaftliche Handlungskriterien anwenden dürfen, denn dann würden sie entweder zur schlechten Kopie oder zur subventionierten 
Konkurrenz von im Markt stehenden Unternehmen. Handeln sie andererseits allgemeinwohlorientiert, arbeiten sie defizitär (MAYER, H.-N. 2005). Aufgrund dieser Zwickmühle kann die Managementrolle nur in Ausnahmefällen und zeitlich befristet ausdifferenziert werden.

\subsection{Kooperationspartner: Planung als strategische Netzwerkgestaltung}

Die Rolle des Kooperationspartners reflektiert stärker den politischen Charakter von Planung und verschneidet ihn mit dem eher klassischen Verständnis einer auf Verstetigung setzenden Planung. Politisch ist diese Rollenauffassung, da Akteure der öffentlichen Verwaltung als Spieler in macht- und interessengeleiteten Konstellationen von Governance (MAYNTZ 2005) auftreten, Allgemeinwohlinteressen definieren und vertreten. Sie zielt auf die Verstetigung von Planaussagen, da Rahmenbedingungen für das künftige Handeln privater Akteure mit den Betroffenen ausgehandelt werden (MAYER, S. 1999; SCHARPF 1991).

Entscheidungsprämissen werden nicht allgemein und von der planenden Verwaltung alleine gestaltet, sondern in ausgewählten Einzelfällen an Verhandlungsrunden delegiert, in denen neben Politik und Verwaltung vor allem die privaten Akteure eingebunden sein müssen, deren Verhalten koordiniert werden soll. Indem die wesentlichen Beteiligten in den Verhandlungen anwesend sind, kann das Zusammenspiel von Entscheidungsprämissen und operationalen Entscheidungen in der Verhandlungssituation durchgespielt und so der Bruch zwischen Planung und Umsetzung überbrückt werden. Dieses Vorgehen folgt der politischen Rationalität, den Raum des «Konsensfähigen» auszureizen und das «Machbare» auch durchzusetzen. Der Umstand, dass öffentliche Akteure die Option der hoheitlichen Steuerung nicht wahrnehmen, obwohl sie sie wahrnehmen könnten, wird als «Faustpfand» in die Verhandlungen eingebracht. Er muss ihnen durch inhaltliche Zugeständnisse und generelle Kooperationsbereitschaft seitens der privaten Akteure erst abgehandelt werden (SCHARPF 1991).

Politische Rationalität lässt Planerinnen und Planer «konspirativ» (RITTEL 1992) werden. Das Entscheiden über Entscheidungen richtet sich auf die Ausgestaltung institutioneller und persönlicher Netzwerke, in denen Verbündete mobilisiert werden. Die operative Projektgruppe, die an der Entwicklung des Gewerbeparks Erin arbeitete, wurde von einem so genannten Patenkreis im Hintergrund unterstützt, einer informellen Runde, in der bis zu dreissig für das Projekt wesentliche öffentliche und private Entscheidungsträger versammelt waren. Solange operative Entscheidungen der Projektgruppe sich in den Entwicklungskorridoren bewegten, die im Patenkreis informell ausgehandelt wurden, war die schnelle Umsetzung gewährleistet.

Wohlfahrtsgewinne ergeben sich, da in kooperativen Verhandlungen oft strategisches Machtverhalten zugunsten fachlich erstrebenswerter Lösungen abgelegt werden kann (MAYNTZ 1993). Die Gewerbeparkentwicklung Erin profitierte davon, dass Investoren von den Qualitäten überzeugt werden konnten und sich bei Investitionen in die eigenen Gebäude, die ja die Standortqualität mitprägen, auf «weiche» ökonomische Kalküle einließen (etwa Imageeffekte). Auch im Patenkreis wurden die Beteiligten auf eine bestmögliche Lösung in diesem speziellen Fall eingeschworen, was dazu führte, dass routineartige Konfrontationen zwischen Interessenvertretern vermieden werden konnten. So erzielen Verhandlungsrunden häufig Lösungen der «positiven Koordination» (SCHARPF 1991), die Verluste und Gewinne so verteilen, dass für die Verhandlungsrunde insgesamt ein positiver Saldo entsteht.

Die Grenzen dieser Rationalität liegen in ihrer Selektivität. Kontroverse Positionen, Grundsatzkonflikte und politische Strategien der Verhinderung lassen sich nicht in kooperative Verhandlungen einbinden (MAYER, M. 2005). Zudem bleiben Kooperationen auf ein mittleres Machtniveau begrenzt. Verhandlungsrunden müssen Entscheidungsmacht konzentrieren, ansonsten drohen Verhandlungsergebnisse ohne Konsequenzen zu bleiben. Gleichwohl ist es auch wichtig, dass die Beteiligten so machtlos sind, dass sie nur gemeinsam ein übergeordnetes Ziel erreichen können. Im Patenkreis Erin fehlten große Konzerne, die in der Lage gewesen wären, ihre Interessen auch ohne Kooperationspartner durchzusetzen. Ebenso fehlten Vertreter marginalisierter Gruppen, etwa der Arbeitslosen in Castrop-Rauxel, da sie zur Problemlösung verzichtbar erschienen.

\subsection{Moderator: Planung als Prozessgestaltung}

Die Moderatorenrolle kombiniert die politische Auffassung von Planung mit einem weitgehenden Verzicht auf die Gestaltung verbindlicher Entscheidungsprämissen. Der politische Charakter dieser Rolle offenbart sich in ihrer Janusköpfigkeit. Nach innen muss die Moderatorenrolle äußerst machtsensibel agieren. Der Einsatz von Macht ist nur möglich, wenn er vom Neutralitätsideal des Moderators gleichsam erzwungen wird. Plannerinnen und Planer müssen Partei ergreifen (BAuHardt 2004: 190), wenn die labile Machtbalance in der moderierten Runde bedroht ist, und zwar zugunsten der schwächeren Parteien. Nach außen müssen Moderatoren dagegen machtbewusst agieren, ihre hierarchischen Befugnisse und ihr politisches und soziales Kapital ausspielen, um die Freiräume zu eröffnen, in denen dann Entscheidungen unter Betroffenen frei ausgehandelt werden. Der Aspekt der Revidier- 
barkeit wird akzentuiert, weil das inhaltliche Ergebnis einer ergebnisoffenen Aushandlungssituation (SELLE 2000) überlassen wird.

Planung wird in dieser Spielart als Prozessgestaltung interpretiert (SELLE 2000). Entscheidungsprämissen sind zu formulieren hinsichtlich der Adressaten diskursiver Prozesse und der Kommunikationsformen (SINNING 2005). Das Umsetzungsdilemma von Planung wird umgangen, indem informell Entscheidungsprämissen vereinbart werden. Im Erfolgsfall fühlen sich die Beteiligten in ihren operativen Entscheidungen diesen Prämissen verpflichtet. Formelle Planungsinstrumente protokollieren diskursiv ausgehandelte Ergebnisse und schaffen so zusätzlich rechtliche Verbindlichkeit. Diese Spielart moderner Planung vertraut auf die Rationalität des Diskurses (Habermas 1981). Prozesse, die im klassischen Planungsmodell in die Figur des planenden Subjekts internalisiert sind, werden einem argumentativen Prozess übertragen, der Probleme aufdeckt und Lösungen zutage fördert (RITTEL 1992).

Innovationsorientierte Verfahren nutzen diese Rolle, etwa wenn es darum geht, das Wissen von Nutzern für Innovationsprozesse $\mathrm{zu}$ mobilisieren. Wenn, wie im IBA-Projekt «Alternatives Wohnen», speziell für die Bedürfnisse allein erziehender Eltern gebaut werden soll, dann fehlt den Experten häufig eine klare Vorstellung der tatsächlichen Bedürfnisse. Moderierter Partizipation kommt hier die Aufgabe zu, das in Alltagserfahrungen verborgene Nutzerwissen zu aktivieren und in die Entwicklung zu integrieren, etwa durch Wohnworkshops und Treffen zwischen Architekten und zukünftigen Nutzern.

Die diskursive Rationalität basiert auf der kontrafaktischen Annahme eines herrschaftsfreien Diskurses. Der Idealzustand muss in der Praxis durch Machtakte gegen bestehende Machtverhältnisse abgesichert werden. Daher ist diskursive Rationalität schwer realisierbar, wenn die öffentliche Hand nicht über Umweltkontrolle verfügt, und zugleich hinreichend Gründe existieren, die Ausübung dieser Kontrolle an ein diskursives Verfahren zu delegieren. Beides war in Recklinghausen der Fall; durch die Grundstückskontrolle, die Sondermittel der IBA sowie die politische Unterstützung durch die Landesregierung wurde ein hohes Maß an Umweltkontrolle erreicht. Durch die Bereitschaft einer Gruppe allein erziehender Eltern, am Experiment aktiv mitzuwirken, existierte ein Nutzerwissen, für das es keine gleichwertige Alternative gab.

\section{Die Grenzen von Strategiefähigkeit}

Komplexe Planungsstrategien sind vorzugsweise möglich, wenn sich das «planende Subjekt» des-inte- griert. Das heißt mehr als bloß das Hin- und Hergerissensein zwischen verschiedenen Rollen, wie es den Planungsalltag seit jeher kennzeichnet. Vielmehr müssen die verschiedenen Rollen deutlich organisatorisch und personell arbeitsteilig ausdifferenziert werden, sie müssen trotz ihrer Rivalität für eine gewisse Zeit aufrechterhalten werden. Beim Planen sollte es unter dem Gesichtspunkt der Strategiefähigkeit keine absoluten Referenzpunkte für Rationalität geben, da jede der beschriebenen Rationalitäten «in bestimmten Planungssituationen gültig [...], in anderen falsch» (SIEBEL 2006: 13) sein kann. Die Restriktionen und Potentiale der jeweiligen Rollen können nur durch die jeweils anderen Referenzpunkte für Rationalität aufgezeigt werden (WIESENTHAL 1990: 65). Solange die Widersprüchlichkeit dieser Handlungslogiken aufrechterhalten und ausgehalten wird, also ein Mindestmaß an «Ambiguitätstoleranz» (WIESENTHAL 1990) herrscht, lässt sich Rivalität produktiv nutzen.

«Eine Planungsstrategie [...] muss paradox konstruiert sein. Die [...] eigentliche Rationalität der Planung liegt in ihrer Fähigkeit [...], sich gleichsam in der Schwebe zu halten im Spannungsfeld verschiedener Rationalitäten» (Siebel 2006: 17f.).

Strategiefähigkeit wird durch Vervielfältigung von Planungsrollen aktiv betrieben. Beide Fallstudien zeigen, dass zeitweise redundante Strukturen neben der existierenden planenden Bürokratie aufgebaut wurden: Parallelbeauftragungen von mehreren Architektenteams, operative Projektgruppen neben der Verwaltung, Zirkel von politischen Förderern im Hintergrund des Prozesses sowie Partizipationsverfahren, die das gesetzlich vorgeschriebene Mindestmaß überschritten. Auf Dauer lassen sich solche Redundanzen in kaum einer Kommune unterhalten, Strategiefähigkeit kann nur ausnahmsweise und zeitlich befristet realisiert werden. Der Energieaufwand erklärt, warum strategisches Handeln, zumindest im Falle innovationsorientierter Planung, eine Affinität zu Festivals (HäUssermanN \& Siebel 1993) zeigt, denn in einem solchen Zusammenhang gelingt es leichter, die entsprechende Energie - in Form von Ressourcen, Aufmerksamkeit, politischer Priorität, Expertise - zu mobilisieren. Doch Festivals trocknen den Alltag daneben und danach womöglich noch mehr aus. An der IBA Emscher Park wurde kritisiert, dass sie Strategiefähigkeit nur an isolierten Projekten erreichen konnte. Der Alltag wurde im Vergleich dazu noch grauer, nicht zuletzt auch, weil ein Teil der Ressourcen, die auf Modellprojekte gebündelt werden, zuvor noch für die Erledigung von Alltagsaufgaben verfügbar war. Auch gibt es Hinweise, dass Strategiefähigkeit zeitlich negativ nachhallt. In der Begeisterung des Aufbruchs aufgenommene Schulden wollen zurückgezahlt, und in Bauwerken mate- 
rialisierte überambitionierte Ziele müssen langfristig unterhalten werden. Beides schränkt die zukünftige Bewegungsfreiheit ein.

Strategiefähigkeit wird nur im komplementären Zusammenspiel vieler Rationalitäten erreicht. Der mächtige Patenkreis im Hintergrund der Gewerbeflächenentwicklung Erin konnte Konflikte vorab klären und so die Handlungsfähigkeit der operativen Projektgruppe erhöhen. In Recklinghausen-Süd konnte der professionelle Gebäudeentwurf durch Nutzerwissen qualifiziert werden, weil die Design-Teams Feedback aus einem Partizipationsprozess erhalten hatten. Komplementarität stellt sich jedoch nicht automatisch ein. So hat das Stadtplanungsamt Recklinghausen die Projektdynamik genutzt, um sehr schnell einen Bebauungsplan zu erstellen und durch den Rat beschließen $\mathrm{zu}$ lassen. Dieser Bebauungsplan enthielt detaillierte Festsetzungen, die die operationale Projektgruppe bei der Flächenentwicklung später als hinderlich empfand, da sie Verhandlungsspielräume mit Investoren reduzierten und Festlegungen enthielten, die neue Ideen in der Lösungsfindung zu ersticken drohten. Die Multiplikation von Planungsrollen erhöht nicht nur die Chance auf alternative Lösungswege, sondern steigert auch die Gefahr von Abstimmungsproblemen. Reibungen und Konflikte sind sicherlich kaum zu vermeiden. Sie müssen aber nicht unbedingt Symptom für fehlende Koordination sein, sondern können als Mechanismen der Selbstorganisation interpretiert werden.

\section{Literatur}

Bauhardt, C. (2004): Entgrenzte Räume. Zu Theorie und Politik räumlicher Planung. - Wiebaden: VS Verlag für Sozialwissenschaften.

BraYbrooke, D. \& C.E. Lindblom (1972): Zur Strategie der unkoordinierten kleinen Schritte (disjointed incrementalism). - In: Fehl, G., Fester, M. \& N. KuHNERT (Hrsg.): Planung und Information. Materialien zur Planungsforschung. - Gütersloh: Bertelsmann: 139-166.

FALUdi, A. (1985): A decision-centred view of environmental planning. - In: Landscape Planning 12: 239256.

FALUdI, A. (1986): Flexibility in US zoning: a European perspective. - In: Environment \& Planning B: Planning and Design 13:255-278.

FARWER, H. (2002): Konstruierte Nachbarschaft. Empirische Evaluation des Projektes «Alternatives Wohnen» in Recklinghausen-Süd im Rahmen der Internationalen Bauausstellung Emscher Park. - Unveröffentlichte Diplomarbeit, Universität Oldenburg, Fachbereich Sozialwissenschaften.

FunKE, R. (1974): Organisationsstrukturen planender Verwaltung. - Schriftenreihe des Bundesministeriums für Raumordnung, Bauwesen und Städtebau, Städte- bauliche Forschung 27, Bonn-Bad Godesberg: Selbstverlag.

Gans, H.J. (1969): Memorandum zur Gesellschaftsplanung. - In: Stadtbauwelt 51/52: 252-256.

Habermas, J. (1981): Theorie des kommunikativen Handelns. Bd. 1-2. - Frankfurt am Main: Suhrkamp.

Häussermann, H. \& W. Siebel (Hrsg.) (1993): Festivalisierung der Stadtpolitik. Stadtentwicklung durch große Projekte. - Leviathan Sonderheft 13, Wiesbaden: Westdeutscher Verlag.

IBERT, O. (2003): Innovationsorientierte Planung. Verfahren und Strategien zur Organisation von Innovation. - Stadt, Raum und Gesellschaft 19, Opladen: Leske + Budrich.

Ibert, O., Mayer, H.-N. \& W. Siebel (2002): Die Organisation von Innovationen: Neue Formen der Stadtund Regionalplanung. Ein Vergleich von EXPO 2000 Hannover und Internationaler Bauausstellung Emscher Park. - Abschlussbericht des DFG-Forschungsprojekts, Arbeitsgruppe Stadtforschung, Carl von Ossietzky Universität Oldenburg.

Luhmann, N. (1971): Politische Planung. Aufsätze zur Soziologie von Politik und Verwaltung. - Opladen: Westdeutscher Verlag.

MAYer, H.-N. (2005): Projekte als spezifische Organisationsform und als neue Entwicklungsstrategie in der Stadt- und Regionalplanung. Kumulative Habilitationsschrift. - Oldenburg: Universität Oldenburg, Fakultät für Human- und Gesellschaftswissenschaften.

MaYer, M. (2005): Das Konzept des Sozialkapitals in der stadtpolitischen Diskussion. - In: Informationen zur Raumentwicklung 9/10.2005: 589-598.

MAYER, S. (1999): Relationale Raumplanung. Ein institutioneller Ansatz für flexible Regulierung. - Marburg: Metropolis.

Mayntz, R. (1993): Policy-Netzwerke und die Logik von Verhandlungssystemen. - In: HÉRITIER, A. (Hrsg.): Policy-Analyse - Kritik und Neuorientierung. - PVS Sonderheft 24, Opladen: Westdeutscher Verlag: 31-50. MAYNTZ, R. (2005): Governance Theory als fortentwikkelte Steuerungstheorie? - In: SCHUPPERT, G.F. (Hrsg.): Governance-Forschung. Vergewisserung über Stand und Entwicklungslinien. - Baden-Baden: Nomos: 1120.

Ritrel, H.W.J. (1992): Planen - Entwerfen - Design. Ausgewählte Schriften zu Theorie und Methodik. Herausgegeben von Wolf D. Reuter. - Stuttgart, Berlin, Köln: W. Kohlhammer.

RoBerTs, T. (2002): The seven lamps of planning. - In: Town Planning Review 73:1-9.

ScharpF, F.W. (1991): Die Handlungsfähigkeit des Staates am Ende des 20. Jahrhunderts. - In: Politische Vierteljahresschrift 4/1991: 621-634.

Selle, K. (2000): Was? Wer? Wie? Warum? Voraussetzungen und Möglichkeiten einer nachhaltigen Kommunikation. - Dortmund: Dortmunder Vertrieb für Bau- und Planungsliteratur. 
Selle, K. (2005): Planen. Steuern. Entwickeln. Über den Beitrag öffentlicher Akteure zur Entwicklung von Stadt und Land. - Dortmund: Dortmunder Vertrieb für Bau- und Planungsliteratur.

Siebel, W. (2006): Wandel, Rationalität und Dilemmata der Planung. - PND Ausgabe 4/2006, www.planung-neu-denken.de 15.08.2007.

SinNing, H. (2005): Partizipation - neue Anforderungen an eine bewährte Governanceform. - In: Informationen zur Raumentwicklung 9/10.2005: 579-588.

SinNING, H. (2006): Stadtmanagement - konzeptionelle Einordnung, Handlungsebenen und Handlungsfelder. - In: SinNING, H. (Hrsg.): Stadtmanagement. Strategien zur Modernisierung der Stadt(-Region). - Dortmund: Dortmunder Vertrieb für Bau- und Planungsliteratur: 132-143.

Storbeck, D. (1969): Zielkonflikt-Systeme als Ansatz zur rationalen Gesellschaftspolitik. Methodologische Überlegungen zur Theorie der Sozial- und Wirtschaftspolitik. - In: Schelsky, H. (Hrsg.): Zur Theorie der allgemeinen und der regionalen Planung. Beiträge zur Raumplanung. - Bielefeld: Bertelsmann: 61-84.

Suchman, L. (1987): Plans and situated actions. The problem of human machine interaction. - New York, Melbourne: Cambridge University Press.

Wiechmann, T. \& G. Hutter (2008): Die Planung des Unplanbaren. Was kann die Raumplanung von der Strategieforschung lernen? - In: Hamedinger, A., Frey, O., Dangschat, J. \& A. Breitfuss (Hrsg.): Strategieorientierte Planung im kooperativen Staat. - Wiesbaden: VS Verlag für Sozialwissenschaften: 102-121.

Wiesenthal, H. (1990): Unsicherheit und MultipleSelf-Identität. Eine Spekulation über die Voraussetzungen strategischen Handelns. - MPIfG Discussion Paper 90/2, Köln: Max-Planck-Institut für Gesellschaftsforschung, http://www.mpi-fg-koeln.mpg.de/pu/ dp88-92_de.asp 15.08.2007.

\section{Zusammenfassung: Von «der» Planung zu multip- len Planungen: Strategische Entscheidungen unter Bedingungen hoher Komplexität und geringer Umweltkontrolle}

Planung bedeutet über Entscheidungen entscheiden. Strategisch kann Planung dann genannt werden, wenn viele Entscheidungen über einen längeren Zeitraum einem Muster folgen und dabei komplexe Ziele realisierbar werden. Strategiefähigkeit ist eine Eigenschaft, die nur kohärenten Akteuren zugeschrieben werden kann. Unter Bedingungen großer externer Unsicherheit und geringer Umweltkontrolle allerdings kann Strategiefähigkeit nur aufrechterhalten werden, wenn es gelingt, nach mehr als nur einer Rationalität vorgehen zu können. Anhand zweier Fallbeispiele werden verschiedene Rollen unterschieden, aus denen heraus geplant werden kann - Ingenieur, Manager, Kooperationspartner und Moderator -, und jeweils die zugrunde liegenden Rationalitätskonzepte, die Organisation von Entscheidungssequenzen sowie instrumentelle Begrenzungen und Potentiale herausgearbeitet. Abschließend werden die Grenzen einer Planung diskutiert, die Strategiefähigkeit durch eine Ausdifferenzierung verschiedener Logiken erreicht.

Schlüsselwörter: Strategische Planung, Entscheidungsprozesse, innovationsorientierte Planung, Internationale Bauausstellung Emscher Park

Summary: From «planning subject» to «multiple-self planning»: strategic decision making under conditions of high complexity and low environmental control

Planning is closely related to decision making. Planning may be considered strategic when several decisions made over a long span of time follow a particular pattern and lead to the fulfillment of complex goals. Strategic capacity is a characteristic commonly accredited to an internally coherent actor, the planning subject. However, under conditions of external uncertainty and low environmental control, strategic capacity can only be upheld when it becomes possible to follow more than one mode of rationality. Use is made of two case studies to identify four distinct roles for planning (engineer, manager, cooperation partner and moderator) and their respective underlying modes of rationality. In conclusion, the limitations of the proposed multiple-self approach towards planning are discussed.

Keywords: strategic planning, processes of decision making, innovation-oriented planning, International Building Exhibition Emscher Park

\section{Résumé: De «la» planification aux planifications: décisions stratégiques dans des conditions de grande complexité et de faible contrôle de l'environnement}

La planification nécessite de prendre des décisions. Elle peut être appelée stratégique lorsqu'un grand nombre de décisions prises sur une longue période de temps suivent un modèle et aboutissent à la réalisation d'objectifs complexes. La capacité stratégique constitue une propriété qui ne peut être attribuée qu'à des acteurs cohérents. Dans des conditions de grande insécurité externe et d'un faible contrôle de l'environnement, la capacité stratégique ne peut être maintenue qu'à la condition d'une forte rationalité. En se fondant sur deux exemples, cet article distingue les différents rôles des acteurs de la planification (ingénieurs, managers, partenaires, modérateurs), les différents concepts de rationalité, l'organisation de la prise de décision, ainsi que les limites et les potentiels identifiés. Enfin, les limites de la planification sont discutées ainsi que la capacité stratégique se basant sur des logiques de différenciation différentes. 
Mots-clés: planification stratégique, processus de prise de décision, planification orientée vers l'innovation, Exposition internationale d'architecture d'Emscher Park

Dr. Oliver Ibert, Sozioökonomie des Raumes, Geographisches Institut, Universität Bonn, Meckenheimer Allee 166, D-53115 Bonn, Deutschland.

e-mail: ibert@giub.uni-bonn.de

\section{Manuskripteingang/received/manuscrit entré le} 17.9.2008

Annahme zum Druck/accepted for publication/accepté pour l'impression: 20.5.2009 